\title{
On the Optimal Parameter Choice for Elliptic Curve Cryptosystems Using Isogeny
}

\author{
Toru Akishita $^{\star 1}$ and Tsuyoshi Takagi ${ }^{2}$ \\ 1 Sony Corporation, Ubiquitous Technology Laboratories, \\ 6-7-35 Kitashinagawa Shinagawa-ku, Tokyo, 141-0001 Japan \\ akishita@pal.arch.sony.co.jp \\ 2 Technische Universität Darmstadt, Fachbereich Informatik, \\ Alexanderstr.10, D-64283 Darmstadt, Germany \\ ttakagi@cdc.informatik.tu-darmstadt.de
}

\begin{abstract}
The isogeny for elliptic curve cryptosystems was initially used for the efficient improvement of order counting methods. Recently, Smart proposed the countermeasure using isogeny for resisting the refined differential power analysis by Goubin (Goubin's attack). In this paper, we examine the countermeasure using isogeny against zero-value point (ZVP) attack that is generalization of Goubin's attack. We show that some curves require higher order of isogeny to prevent ZVP attack. Moreover, we prove that this countermeasure cannot transfer a class of curve to the efficient curve that is secure against ZVP attack. This class satisfies that the curve order is odd and $(-3 / p)=-1$ for the base field $p$, and includes three SECG curves. In the addition, we compare some efficient algorithms that are secure against both Goubin's attack and ZVP attack, and present the most efficient method of computing the scalar multiplication for each curve from SECG. Finally, we discuss another improvement for the efficient scalar multiplication, namely the usage of the point $(0, y)$ for the base point of curve parameters. We are able to improve about $11 \%$ for double-and-add-always method, when the point $(0, y)$ exists in the underlying curve or its isogeny.
\end{abstract}

Keywords: elliptic curve cryptosystems, isomorphism, isogeny, side channel attack, zero-value point attack.

\section{Introduction}

Elliptic curve cryptosystem (ECC) is an efficient public-key cryptosystem with a short key size. ECC is suitable for implementing on memory-constraint devices such as mobile devices. However, if the implementation is careless, side channel attack (SCA) might reveal the secret key of ECC. We have to carefully investigate the implementation of ECC in order to achieve the high security.

The standard method of defending SCA on ECC is randomizing the curves parameters, for instance, randomizing a base point in projective coordinates [5]

* This work was done while the first author stayed at Technische Universität Darmstadt, Germany. 
and randomizing curve parameters in the isomorphic class [11]. However, Goubin pointed out that the point $(0, y)$ cannot be randomized by these methods [7]. He proposed a refined differential power analysis using the point $(0, y)$. This attack has been extended to the zero value of the auxiliary registers, called the zerovalue point (ZVP) attack [1]. Both Goubin's attack and the ZVP attack assume that the base point $P$ can be chosen by the attacker and the secret scalar $d$ is fixed, so that we need to care these attacks in ECIES and single-pass ECDH, but not in ECDSA and two-pass ECDH.

In order to resist Goubin's attack, Smart proposed to map the underlying curve to the isogenous curve that does not have the point $(0, y)[17]$. This countermeasure with a small isogeny degree is faster than randomizing the secret scalar $d$ with the order of the curve. However, the security of this countermeasure against the ZVP attack has not been discussed yet - it could be vulnerable to the ZVP attack.

\subsection{Contribution of This Paper}

In this paper, we examine the countermeasure using isogeny against the ZVP attack. The zero-value points (ED1) $3 x^{2}+a=0,(M D 1) x^{2}-a=0$, and (MD2) $x^{2}+a=0$ were examined. We show that some curves require higher order of isogeny to prevent the ZVP attack. For example, SECG secp112r1 [18] is secure against Goubin's attack, but insecure against the ZVP attack. Then, the 7isogenous curve to secp112r1 is secure against both attacks. We require isogeny of degree 7 to prevent the ZVP attack. For each SECG curve we search the minimal degree of isogeny to the curve that is secure against both Goubin's attack and the ZVP attack. Since the ZVP attack strongly depends on the structure of addition formula, the minimal degree of isogeny depends on not only the curve itself but also addition formula. Interestingly, three SECG curves cannot be mapped to the curve with $a=-3$ that is secure against the ZVP attack. The curve with $a=-3$ is important for efficiency. We prove that this countermeasure cannot map a class of curve to the curve with $a=-3$ that is secure against the ZVP attack. This class satisfies that the curve order is odd and $(-3 / p)=-1$ for the base field $p$, and these three curves belong to this class.

Moreover, we estimate the total cost of the scalar multiplication in the necessity of resistance against both Goubin's attack and the ZVP attack. We compare two efficient DPA-resistant methods, namely the window-based method and Montgomery-type method, with the countermeasure using isogeny, and present the most efficient method to compute the scalar multiplication for each SECG curve.

Finally we show another efficient method for computing the scalar multiplication, namely using the point $(0, y)$ for the base point. We can prove the discrete logarithm problem with the base point $(0, y)$ is as intractable as using a random one thanks to the random self reducibility. Comparing with the previous method we are able to achieve about $11 \%$ faster scalar multiplication using the double-and-add-always method. This base point can also save 50\% memory space without any compression trick. We propose the scenario to utilize 
the proposed method efficiently and show the example of a curve to achieve this scenario.

This paper is organized as follows: Section 2 briefly reviews known results about elliptic curve cryptosystems. Section 3 describes the choices of secure curve against the ZVP attack using isogeny. In Section 4 we show the efficient implementations using isogeny. In Section 5 we state concluding remarks.

\section{Elliptic Curve Cryptosystems}

In this section we review some results on elliptic curve cryptosystems related to isogeny. Let $K=\mathbb{F}_{p}$ be a finite field, where $p>3$. The Weierstrass form of an elliptic curve over $K$ is described as

$$
E: y^{2}=x^{3}+a x+b \quad\left(a, b \in K, \Delta=-16\left(4 a^{3}+27 b^{2}\right) \neq 0\right) .
$$

The set of all points $P=(x, y)$ satisfying $E$, together with the point of infinity $\mathcal{O}$, is denoted by $E(K)$, which forms an Abelian group. Let $P_{1}=\left(x_{1}, y_{1}\right)$ and $P_{2}=\left(x_{2}, y_{2}\right)$ be two points on $E(K)$ that don't equal to $\mathcal{O}$. The sum $P_{3}=$ $P_{1}+P_{2}=\left(x_{3}, y_{3}\right)$ can be computed as $x_{3}=\lambda\left(P_{1}, P_{2}\right)^{2}-x_{1}-x_{2}, \quad y_{3}=$ $\lambda\left(P_{1}, P_{2}\right)\left(x_{1}-x_{3}\right)-y_{1}$, where $\lambda\left(P_{1}, P_{2}\right)=\left(3 x_{1}^{2}+a\right) /\left(2 y_{1}\right)$ for $P_{1}=P_{2}$, and $\lambda\left(P_{1}, P_{2}\right)=\left(y_{2}-y_{1}\right) /\left(x_{2}-x_{1}\right)$ for $P_{1} \neq \pm P_{2}$. We call the former, $P_{1}+P_{2}\left(P_{1}=\right.$ $\left.P_{2}\right)$, the elliptic curve doubling (ECDBL) and the latter, $P_{1}+P_{2}\left(P_{1} \neq \pm P_{2}\right)$, the elliptic curve addition (ECADD) in affine coordinate $(x, y)$. These two addition formulae respectively need one inversion over $K$, which is much more expensive than multiplication over $K$. Therefore, we transform affine coordinate $(x, y)$ into other coordinates where inversion is not required. In this paper we deal with Jacobian coordinates $(X: Y: Z)$ setting $x=X / Z^{2}$ and $y=Y / Z^{3}$. The doubling and addition formulae can be represented as follows.

ECDBL in Jacobian Coordinates $\left(\mathrm{ECDBL}^{\mathcal{J}}\right)$ :

$X_{3}=T, Y_{3}=-8 Y_{1}^{4}+M(S-T), Z_{3}=2 Y_{1} Z_{1}$, $S=4 X_{1} Y_{1}^{2}, M=3 X_{1}^{2}+a Z_{1}^{4}, T=-2 S+M^{2}$.

ECADD in Jacobian Coordinates $\left(\mathrm{ECADD}^{\mathcal{J}}\right)$ :

$X_{3}=-H^{3}-2 U_{1} H^{2}+R^{2}, Y_{3}=-S_{1} H^{3}+R\left(U_{1} H^{2}-X_{3}\right), Z_{3}=Z_{1} Z_{2} H$,

$U_{1}=X_{1} Z_{2}^{2}, U_{2}=X_{2} Z_{1}^{2}, S_{1}=Y_{1} Z_{2}{ }^{3}, S_{2}=Y_{2} Z_{1}^{3}, H=U_{2}-U_{1}, R=S_{2}-S_{1}$.

We call these formulae as the standard addition formulae. For ECADD ${ }^{\mathcal{J}}$ we require 16 multiplications when $Z_{1} \neq 1$ and 11 ones when $Z_{1}=1$. For ECDBL E $^{\mathcal{H}}$ we require 10 multiplications in general, 9 ones when $a$ is small, and only 8 ones when $a=-3$ by $M=3\left(X_{1}+Z_{1}^{2}\right)\left(X_{1}-Z_{1}^{2}\right)$. Thus all SECG random curves over $\mathbb{F}_{p}$ with prime order satisfy $a=-3$. In this paper, we are interested in the curves with prime order such as these curves.

\subsection{Scalar Multiplication and Side Channel Attack}

The scalar multiplication evaluates $d P$ for a given integer $d$ and a base point $P$ of ECC. A standard algorithm of computing $d P$ is a binary method, which is implemented by repeatedly calling ECDBL and ECADD. Let $d=\left(d_{n-1} \cdots d_{1} d_{0}\right)_{2}$ 
be the binary representation of $d$ where $d_{n-1}=1$ The binary method is as follows:
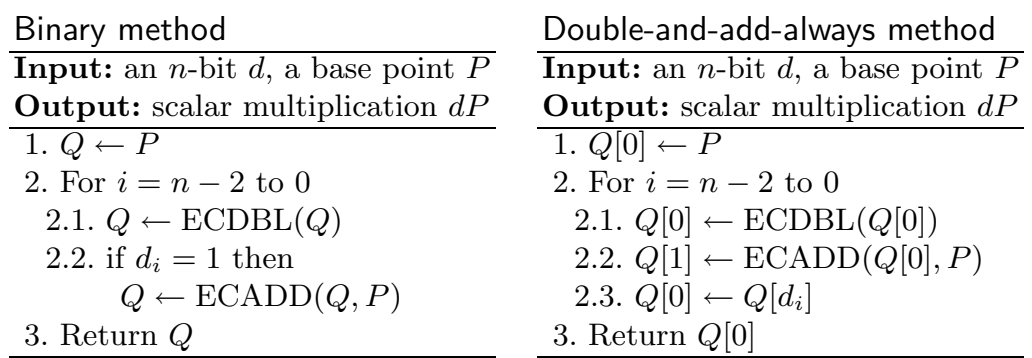

The SPA uses a single observation of the power consumption to obtain the information of secret key. The binary method is vulnerable to SPA. Since ECADD is computed only if the underlying bit is 1 and a SPA attacker can distinguish ECDBL and ECADD, he can detect the secret bit. Coron proposed a simple countermeasure called as the double-and-add-always method [5]. The attacker cannot guess the bit information because this method always computes ECADD whether $d_{i}=0$ or 1 . Two more efficient methods have been proposed. The first is window-based method $[13,14,16]$ and the second is Montgomery-type method [3, $6,8-10]$.

The DPA uses many observations of the power consumption together with statistical tools. To enhance SPA security to DPA security, we must insert random numbers during computation of $d P$. The standard randomization methods for the base point $P$ are Coron's 3rd countermeasure [5] and Joye-Tymen countermeasure [11]. In order to randomize the representation of the processing point, Coron's 3rd countermeasure uses randomized representation of Jacobian (projective) coordinates and Joye-Tymen countermeasure uses randomized isomorphism of an elliptic curve.

\subsection{Efficient Method Secure against DPA}

Window-based Method The window-based method secure against SPA was first proposed by Möller [13,14], and optimized by Okeya and Takagi [16]. This method uses the standard addition formulae the same as the double-and-addalways method. It makes the fixed pattern $|0 \cdots 0 x| 0 \cdots 0 x|\cdots| 0 \cdots 0 x \mid$ for some $x$. Though the SPA attacker distinguishes ECDBL and ECADD in the scalar multiplication by measuring the power consumption, he obtains only the identical sequence $|D \cdots D A| D \cdots D A|\cdots| D \cdots D A \mid$, where $D$ and $A$ denote ECDBL and ECADD, respectively. Therefore, he cannot guess the bit information. This method reduces ECADD as compared with the double-and-add-always method and thus enables efficiency. In order to enhance this method to be DPA-resistant, we have to insert a random value using Coron's 3rd countermeasure or JoyeTymen countermeasure. Moreover, we have to randomize the value of table to protect 2nd order DPA. We estimate the computational cost of the scalar multiplication $d P$ according to [16]. Denote the computational cost of multiplica- 
tion and inversion in the definition field by $M$ and $I$, respectively. The total cost is estimated as $\left.\left(16 \cdot 2^{w}+(9 w+21) k-18\right)\right) M+I$ when $a$ is small and $\left.\left(16 \cdot 2^{w}+(8 w+21) k-18\right)\right) M+I$ when $a=-3$, where $n$ is the bit length of $d$, $w$ is the window size, and $k=\lceil n / w\rceil$.

Montgomery-Type Method Montgomery-type method was originally proposed by Montgomery [15] and enhanced to the Weierstrass form of elliptic curves over $K[3,6,8-10]$. This method always computes ECADD and ECDBL whether $d_{i}=0$ or 1 as the double-and-add-always method, and thus satisfies SPA-resistance. In this method, we don't need to use $y$-coordinate ( $Y$-coordinate in projective coordinates) to compute the scalar multiplication $d P$. This leads the efficiency of Montgomery-type method. In the original method ECADD and ECDBL are computed separately. However, Izu and Takagi encapsulated these formulae into one formula mECADDDBL to share intermediate variables and cut two multiplications [10]. Let $P_{1}=\left(X_{1}: Z_{1}\right)$ and $P_{2}=\left(X_{2}: Z_{2}\right)$ in projective coordinates, which don't equal to $\mathcal{O}$, by setting $x=X / Z$. In the following we describe the encapsulated formula mECADDDBL ${ }^{\mathcal{P}}$, which compute $P_{3}=\left(X_{3}: Z_{3}\right)=P_{1}+P_{2}$ and $P_{4}=\left(X_{4}: Z_{4}\right)=2 P_{1}$, where $P_{1} \neq \pm P_{2}$, $P_{3}{ }^{\prime}=\left(X_{3}{ }^{\prime}: Z_{3}{ }^{\prime}\right)=P_{1}-P_{2}$ and $\left(X_{3}^{\prime}, Z_{3}^{\prime} \neq 0\right)$.

ECADDDBL in Montgomery-Type Method (mECADDDBL ${ }^{\mathcal{P}}$ ) :

$\left.X_{3}=Z_{3}{ }^{\prime}\left(2\left(X_{1} Z_{2}+X_{2} Z_{1}\right)\left(X_{1} X_{2}+a Z_{1} Z_{2}\right)+4 b Z_{1}{ }^{2} Z_{2}{ }^{2}\right)\right)-X_{3}{ }^{\prime}\left(X_{1} Z_{2}-X_{2} Z_{1}\right)^{2}$, $Z_{3}=Z_{3}{ }^{\prime}\left(X_{1} Z_{2}-X_{2} Z_{1}\right)^{2}$

$X_{4}=\left(X_{1}^{2} Z_{2}^{2}-a Z_{1}^{2} Z_{2}^{2}\right)^{2}-8 b X_{1} Z_{1}^{3} Z_{2}^{4}$,

$Z_{4}=4 Z_{1} Z_{2}\left(X_{1} Z_{2}\left(X_{1}^{2} Z_{2}^{2}+a Z_{1}^{2} Z_{2}^{2}\right)+b Z_{1}^{3} Z_{2}^{3}\right)$.

We call this formula as Montgomery-type addition formula. mECADDDBL requires 17 multiplications in general and 15 ones when $a$ is small. In order to enhance this method to DPA-resistant, we have to use Coron's 3rd countermeasure or Joye-Tymen countermeasure. The total cost of scalar multiplication $d P$ is estimated as $(17 n+8) M+I$ in general and $(15 n+10) M+I$ when $a$ is small, where $n$ is the bit length of the scalar $d$ (see [8]).

\subsection{Isomorphism and Isogeny}

Two elliptic curves $E_{1}\left(a_{1}, b_{1}\right)$ and $E_{2}\left(a_{2}, b_{2}\right)$ are called isomorphic if and only if there exists $r \in K^{*}$ such that $a_{1}=r^{4} a_{2}$ and $b_{1}=r^{6} b_{2}$. The isomorphism is given by

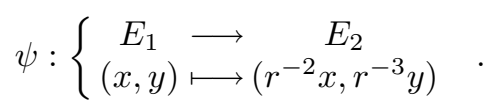

There are $(p-1) / 2$ isomorphic classes.

Let $\Phi_{l}(X, Y)$ be a modular polynomial of degree $l$. Two elliptic curves $E_{1}\left(a_{1}, b_{1}\right)$ and $E_{2}\left(a_{2}, b_{2}\right)$ are called $l$-isogenous if and only if $\Phi_{l}\left(j_{1}, j_{2}\right)=0$ satisfies, where $j_{i}$ are $j$-invariant of curve $E_{i}$ for $i=1,2$. Isogenous curves have the same order. 
The isogeny is given by

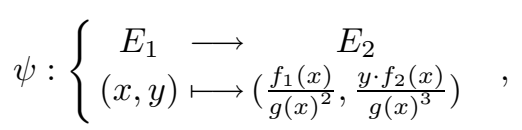

where $f_{1}, f_{2}$ and $g$ are polynomials of degree $l,(3 l-1) / 2$ and $(l-1) / 2$ respectively (see details in $[2$, Chapter VII]). By Horner's rule, the computational cost of this mapping is estimated as $(l+(3 l-2) / 2+(l-1) / 2+5) M+I=(3 l+4) M+I$.

The usage of isogeny for elliptic curve cryptosystem initially appeared for improving the order counting method (see, for example, [12]). Recently, some new applications of isogeny have been proposed, namely for improving the efficiency of the scalar multiplication [4], and for enhancing the security for a new attack [17].

Brier and Joye reported that isogeny could be used for improving the efficiency of ECDBL ${ }^{\mathcal{J}}$ [4]. Recall that if the curve parameter $a$ of an elliptic curve is equal to -3 , the cost of $\mathrm{ECDBL}^{\mathcal{J}}$ is reduced from 10 multiplications to 8 ones. If there is an integer $r$ such that $-3=r^{4} a$, then we can transform the original elliptic curve to the isomorphic curve with $a=-3$. However, its success probability is about $1 / 2$ when $p \equiv 3 \quad(\bmod 4)$ or about $1 / 4$ when $p \equiv 1 \quad(\bmod 4)$. They proposed that the isogeny of the original curve could have a curve with $a=-3$.

Goubin proposed the new power analysis on ECC [7]. This attack utilizes the points $(x, 0)$ and $(0, y)$ that cannot be randomized by the above two standard randomization techniques. Goubin's attack is effective on the curves that have point $(x, 0)$ or $(0, y)$ in such protocols as ECIES and single-pass ECDH. The point $(x, 0)$ is not on the curve with prime order because the order of $(x, 0)$ is 2 . The point $(0, y)$ appears on the curve if $b$ is quadratic residue modulo $p$, which is computed by solving $y^{2}=b$. As a countermeasure to Goubin's attack, Smart utilized isogeny [17]. He proposed that if the original curve $E$ has the point $(0, y)$, the isogenous curve $E^{\prime}$ to $E$ could have no point $(0, y)$. If we can find $E^{\prime}$ which has no point $(0, y)$, we transfer the base point $P \in E$ to $P^{\prime} \in E^{\prime}$ using the isogeny $\psi: E \rightarrow E^{\prime}$. Instead of computing scalar multiplication $Q=d P$, we compute $Q^{\prime}=d P^{\prime}$ on $E^{\prime}$ and then pull back $Q \in E$ from $Q^{\prime} \in E^{\prime}$ by the mapping $\psi^{-1}: E^{\prime} \rightarrow E$. The mappings $\psi, \psi^{-1}$ require $(3 l+4) M+I$ respectively, so that the additional cost for this countermeasure is $(6 l+8) M+2 I$.

At ISC'03, we proposed the zero-value point (ZVP) attack which is extension of Goubin's attack [1]. We pointed out that if the point has no zero-value coordinate, the auxiliary registers might take zero-value. We found several points $(x, y)$ which cause the zero-value registers and called these points as the zerovalue points (ZVP). ZVP strongly depend on the structure of addition formula, and namely ZVP for the standard addition formulae are different from those for Montgomery addition formula. The points with the following conditions from ECDBL are effectively used for the ZVP attack.

- (ED1) $3 x^{2}+a=0$ for the standard addition formulae

- (MD1) $x^{2}-a=0$ and (MD2) $x^{2}+a=0$ for Montgomery addition formula 
The attacker can utilize the points that cause the zero-value registers in ECADD, however finding ZVP in ECADD is much more difficult than in ECDBL. In this paper we consider only the above points (ED1), (MD1), and (MD2)).

\section{Isogeny Countermeasure against ZVP Attack}

In this section we examine the countermeasure using isogeny against the ZVP attack. In order to prevent the ZVP attack, we have to choose the curve which has neither the point $(0, y)$ nor (ED1) for the methods using the standard addition formulae, and neither $(0, y)$, (MD1) nor (MD2) for Montgomery-type method. The degree of isogeny depends on not only a curve itself but also addition formulae. We examine the standard curves from SECG [18].

\subsection{Example from SECG Curve}

For example, we mention the curve secp112r1 from SECG curves [18]. secp112r1 $E: y^{2}=x^{3}+a x+b$ over $\mathbb{F}_{p}$ is defined by

$$
\left\{\begin{array}{l}
p=4451685225093714772084598273548427 \\
a=4451685225093714772084598273548424=-3, \\
b=2061118396808653202902996166388514
\end{array}\right.
$$

This curve does not have $(0, y)$, but has (ED1) $3 x^{2}+a=0$ as

$$
(x, y)=(1,1170244908728626138608688645279825) .
$$

Therefore secp112r1 is secure against Goubin's attack, but vulnerable against the ZVP attack for the methods using the standard addition formulae. However, the 7 -isogenous curve $E^{\prime}: y^{2}=x^{3}+a^{\prime} x+b^{\prime}$ over $\mathbb{F}_{p}$ defined by

$$
\left\{\begin{array}{l}
a^{\prime}=1, \\
b^{\prime}=811581442038490117125351766938682,
\end{array}\right.
$$

has neither $(0, y)$ nor ( $E D 1) 3 x^{2}+a^{\prime}=0$. Thus $E^{\prime}$ is secure against both Goubin's attack and the ZVP attack for the methods using the standard addition formulae. We don't require isogeny defense to prevent Goubin's attack, but require the isogeny of degree 7 to prevent the ZVP attack.

\subsection{Experimental Results from SECG Curves}

For each SECG curve we search the minimal degree of isogeny to a curve which has neither $(0, y)$ nor ZVP as described above. If the original curve has neither $(0, y)$ nor ZVP, we specify this degree as 1 . For the standard addition formulae, we also search the minimal isogeny degree to a curve which we prefer for particularly efficient implementation, namely $a=-3$ as described in section 2 . We call the former as the minimal isogeny degree and the latter as the preferred isogeny degree, and define $l_{\mathrm{std}}, l_{\mathrm{prf}}$, and $l_{\mathrm{mnt}}$ as follows: 


\begin{tabular}{|c||c|c|c|}
\hline & $l_{\text {std }}$ & $l_{\text {prf }}$ & $l_{\text {mnt }}$ \\
\hline \hline secp112r1 & $7(1)$ & $>107(1)$ & $1(1)$ \\
\hline secp128r1 & $7(7)$ & $7(7)$ & $7(7)$ \\
\hline secp160r1 & $13(13)$ & $13(13)$ & $19(13)$ \\
\hline secp160r2 & $19(19)$ & $41(41)$ & $19(19)$ \\
\hline secp192r1 & $23(23)$ & $>107(73)$ & $23(23)$ \\
\hline secp224r1 & $1(1)$ & $1(1)$ & $1(1)$ \\
\hline secp256r1 & $3(3)$ & $23(11)$ & $3(3)$ \\
\hline secp384r1 & $31(19)$ & $>107(19)$ & $19(19)$ \\
\hline secp521r1 & $5(5)$ & $5(5)$ & $7(5)$ \\
\hline
\end{tabular}

Table 1. Minimal and preferred isogeny degree for SECG curves

$-l_{\text {std }}$ : the minimal isogeny degree for the standard addition formulae,

$-l_{\text {prf }}:$ the preferred isogeny degree for the standard addition formulae,

$-l_{\mathrm{mnt}}$ : the minimal isogeny degree for Montgomery-type addition formula.

Here we show the searching method of these degrees for the standard addition formulae.

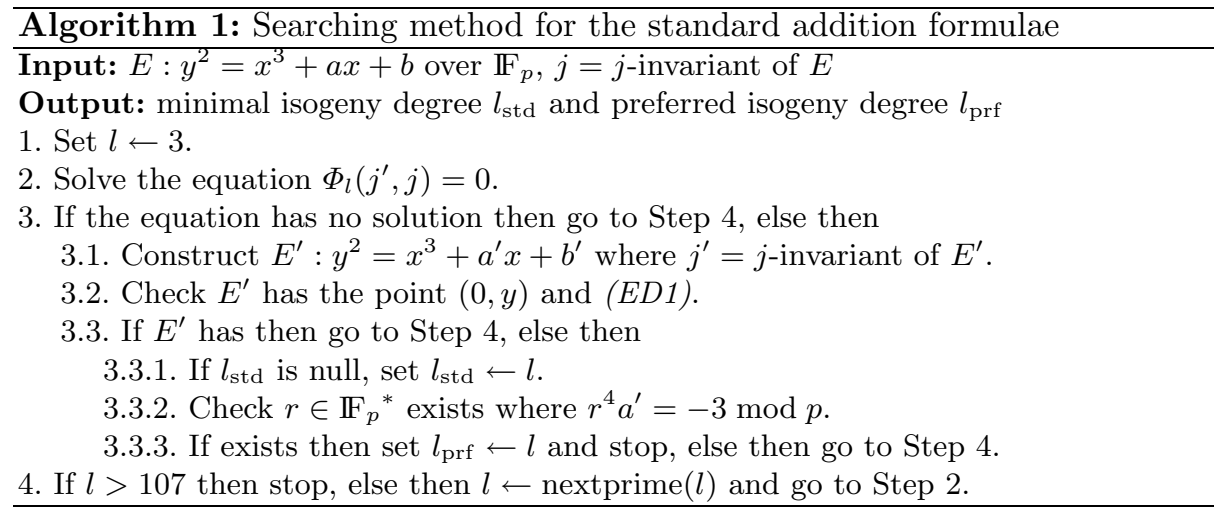

In this algorithm nextprime $(l)$ is a function which returns the smallest prime number larger than $l$. For $l_{\mathrm{mnt}}$, we check (MD1) and (MD2) instead of (ED1) in Step 3.2.

Table 1 shows isogeny degrees $l_{\mathrm{std}}, l_{\mathrm{prf}}$, and $l_{\mathrm{mnt}}$ for SECG curves. The number in $(\cdot)$ is the minimal isogeny degree listed in [17], which considers only Goubin's point $(0, y)$ (not the ZVP). In order to prevent the ZVP attack, some curves require higher degree of isogeny, e.g., secp112r1 for $l_{\text {std }}$. These isogeny degrees depend on not only the curve itself but also the addition formula, namely some curves require different isogeny degrees for the standard addition formulae and Montgomery-type addition formula. Interestingly, we have not found preferred isogeny degree up to 107 for secp112r1, secp192r1, and secp384r1. 


\subsection{Some Properties of ZVP Attack}

Here we show some properties of the zero-value point attack.

Theorem 1. Let $E$ be an elliptic curve over prime field $\mathbb{F}_{p}$ defined by $y^{2}=$ $x^{3}+a x+b$. The elliptic curve $E$ has point $(0, y)$, if $E$ satisfies (MD2) $x^{2}+a=0$.

Proof. If $a=0$ or $b=0$ holds, then the assertion is trivial. We assume that $a \neq 0$ and $b \neq 0$. Note that $(0, y)$ exists on curve $E$ if $b$ is a quadratic residue in $\mathbb{F}_{p}^{*}$. Let $s \in \mathbb{F}_{p}^{*}$ be the solution of equation $x^{2}+a=0$. Condition (MD2) implies that there is a solution $y=t$ of equation $y^{2}=s^{3}+a s+b$. Thus $E$ has point $(0, t)$ due to $t^{2}=s^{3}+a s+b=\left(s^{2}+a\right) a+b=b$.

All curves which satisfy condition (MD2) have Goubin point $(0, y)$. These curves are insecure against both Goubin's attack and the ZVP attack.

Theorem 2. Let $E$ be an elliptic curve over prime field $\mathbb{F}_{p}$ defined by $y^{2}=$ $x^{3}+a x+b$. The elliptic curve $E$ satisfies condition (ED1) $3 x^{2}+a=0$, if $E$ satisfies the following three conditions: (1) $a=-3$, (2)\#E is odd, and (3)p satisfies $(-3 / p)=-1$, where $(\cdot / \cdot)$ is Legendre symbol.

Proof. Since $E$ has odd order, $E$ does not have the point $(x, 0)$, and thus the equation $x^{3}+a x+b=0$ has no root. Then the definition of discriminant $\Delta$ yields $(\Delta / p)=1$. Note that condition $(-3 / p)=-1$ implies $((b+2)(b-2) / p)=-1$ due to $\left.\Delta=-16\left(4(-3)^{3}\right)+27 b^{2}\right)=-3(12)^{2}(b+2)(b-2)$. Thus either $((b+2) / p)=1$ or $((b-2) / p)=-1$ holds. In other words, equation $y^{2}=x^{3}+a x+b$ with $a=-3$ and $x= \pm 1$ are solvable in $y$. Consequently, elliptic curve $E$ with the above three conditions satisfies (ED1) $3 x^{2}+a=0$.

The definition fields $\mathbb{F}_{p}$ that satisfy $(-3 / p)=-1$ in Table 1 are secp112r1, secp192r1, and secp384r1. These curves also have odd order and satisfy $a=-3$. Therefore, these curves satisfy (ED1) and are vulnerable to the ZVP attack.

Since the isogenous curve has same order as $E$, any isogenous curve with $a=-3$ always satisfies $(E D 1)$ and thus is insecure against the ZVP attack. We have the following corollary.

Corollary 1. Let $E$ be an elliptic curve over prime field $\mathbb{F}_{p}$. We assume that $\# E$ is odd and $(-3 / p)=-1$. Any isogeny cannot map $E$ to the curve with $a=-3$ that is secure against the ZVP attack.

Corollary 1 shows that it is impossible to find the isogenous curve with $a=$

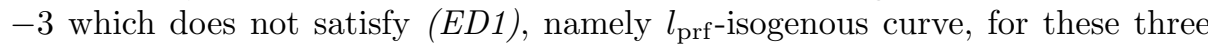
curves.

\section{Efficient Implementation Using Isogeny}

\subsection{Most Efficient Method for Each SECG Curve}

We estimate the total cost of the scalar multiplication in the necessity of resistance against both Goubin's attack and the ZVP attack. This situation corresponds to the scalar multiplication in ECIES and single-pass ECDH. 


\begin{tabular}{|l|c|c|c|}
\hline & Method 1 & Method 2 & Method 3 \\
\hline \hline secp112r1 & $1884 M+3 I(w=4)$ & - & $\mathbf{1 6 9 0 M}+\mathbf{I}$ \\
\hline secp128r1 & $2112 M+3 I(w=4)$ & $1984 M+3 I(w=4)$ & $\mathbf{1 9 8 0 M}+\mathbf{3 I}$ \\
\hline secp160r1 & $2604 M+3 I(w=4)$ & $\mathbf{2 4 4 4 M + 3 I}(\mathbf{w}=\mathbf{4})$ & $2532 M+3 I$ \\
\hline secp160r2 & $2640 M+3 I(w=4)$ & $2612 M+3 I(w=4)$ & $\mathbf{2 5 3 2} \mathbf{M}+\mathbf{3 I}$ \\
\hline secp192r1 & $3120 M+3 I(w=4)$ & - & $\mathbf{3 0 3 6}+\mathbf{3 I}$ \\
\hline secp224r1 & $3430 M+I(w=4)$ & $\mathbf{3 2 0 6} \mathbf{M}+\mathbf{I}(\mathbf{w}=\mathbf{4})$ & $3370 M+I$ \\
\hline secp256r1 & $3912 M+3 I(w=4)$ & $\mathbf{3 7 7 6} \mathbf{M}+\mathbf{3 I}(\mathbf{w}=\mathbf{4})$ & $3876 M+3 I$ \\
\hline secp384r1 & $\mathbf{5 7 7 0 M}+\mathbf{3 I}(\mathbf{w}=\mathbf{5})$ & - & $5892 M+3 I$ \\
\hline secp521r1 & $7462 M+3 I(w=5)$ & $\mathbf{6 9 3 7} \mathbf{M}+\mathbf{3 I}(\mathbf{w}=\mathbf{5})$ & $7875 M+3 I$ \\
\hline
\end{tabular}

Table 2. Total cost of scalar multiplication to resist Goubin's attack and the ZVP attack

Here we notice the two efficient DPA-resistant methods, namely the windowbased method and Montgomery-type method. We have to use the window-based method on $l_{\text {std }}$-isogenous curve because this method uses the standard addition formulae. Isomorphism enables the efficient implementation with small $a$. Moreover, more efficient implementation with $a=-3$ can be achieved on $l_{\text {prf }}{ }^{-}$ isogenous curve. On the other hand, we have to use Montgomery-type method on $l_{\text {mnt }}$-isogenous curve. Isomorphism also enables the efficient implementation with small $a$.

Therefore, we mention the following three methods:

Method 1 Window-based method with small $a$ on $l_{\text {std-isogenous curve, }}$

Method 2 Window-based method with $a=-3$ on $l_{\text {prf }}$-isogenous curve,

Method 3 Montgomery-type method with small $a$ on $l_{\text {mnt-isogenous curve. }}$

From section 2 we estimate the total cost of each method as follows:

Method $1 T_{1}=\left(16 \cdot 2^{w}+(9 w+21) k+6 l_{\mathrm{std}}-10\right) M+3 I$.

Method $2 T_{2}=\left(16 \cdot 2^{w}+(8 w+21) k+6 l_{\text {prf }}-10\right) M+3 I$,

Method $3 T_{3}=\left(15 n+6 l_{\text {mnt }}+18\right) M+3 I$.

If the isogeny degree equals to 1 , the cost of isogeny $(14 M+2 I)$ is cut.

Table 2 shows the estimated cost for each SECG curve. Method 2 cannot be used for some curves because there is no preferred isogeny degree $l_{\text {prf }}$ (notation '_-' indicates these curves). We emphasize the most efficient method for each curve with the bold letter. The most efficient method differs on each curve because the isogeny depends on the curve and implementation method.

\subsection{Efficient Scalar Multiplication Using $(0, y)$}

In this section we propose another improvement for computing the efficient scalar multiplication.

In order to clearly describe our method, we categorize the improvement of efficiency into five classes, namely, (1)curve parameter (e.g. $a=-3, Z=1$, 
etc), (2)addition chain (e.g. binary method, NAF, etc), (3)base field (e.g. optimal normal base, OEF, etc), (4)coordinate (e.g. projective coordinates, Jacobian coordinates, etc). (5)curve form (e.g. Montgomery form, Hessian form, etc). The proposed method belongs to class (1), but its improvement is related to classes (2), (4), and (5). Our improvement can be simultaneously used with other methods in class one. For sake of convenience, we discuss the improvement for the double-and-add-always method in section 2 on the curve with parameter $a=-3, Z=1$, Jacobian coordinate, and Weierstrass form.

The main idea of the improvement is to use the point $(0, y)$ for the base point of the underlying curve, namely the point with the zero $x$-coordinate. The double-and-add-always method in section 2 is a left-to-right method, and thus the base point $P$ is fixed during the scalar multiplication $d P$. The addition formula with the point $X=0$ is represent as follows:

ECADD in Jacobian Coordinates with $X=0\left(\operatorname{ECADD}_{X=0}^{\mathcal{J}}\right)$ : $X_{3}=-H^{3}+R^{2}, Y_{3}=-S_{1} H^{3}-R X_{3}, Z_{3}=Z_{1} Z_{2} H$, $H=X_{2} Z_{1}^{2}, S_{1}=Y_{1} Z_{2}^{3}, S_{2}=Y_{2} Z_{1}^{3}, R=S_{2}-S_{1}$.

We denote by $\operatorname{ECADD}_{X=0}^{\mathcal{J}}$ the addition formula for ECADD in Jacobian Coordinates with $X=0$. Formula $\operatorname{ECADD}_{X=0}^{\mathcal{J}}$ requires only 14 multiplications when $Z_{1} \neq 1$ and 9 multiplications when $Z_{1}=1$.

Therefore, we have the following estimation for $n$-bit scalar multiplication with $a=-3, Z=1$ using Jacobian coordinates and the double-and-add-always method in section 2 . The propose scheme can achieve about $11 \%$ improvement over the scheme $X \neq 0$.

\begin{tabular}{c|c|c}
\hline \hline & $n$-bit ECC & 160-bit ECC \\
\hline Scheme $X \neq 0$ & $19 n M$ & $3040 M$ \\
Scheme $X=0$ & $17 n M$ & $2720 M$ \\
\hline \hline
\end{tabular}

Table 3. Comparison of efficiency with $X \neq 0$ and $X=0$

Here we have a question about the security of choosing the base point $(0, y)$. The following theorem can be easily proven thank to the random self reducibility.

Theorem 3. Let $E$ be an elliptic curve over $\mathbb{F}_{p}$. We assume that $\# E$ is a prime order. Breaking the discrete logarithm problem with base point $(0, y)$ is as intractable as doing with a random base point.

Proof. $(\Leftarrow)$ Let $\log _{G_{0}} P_{0}$ be the discrete logarithm problem for the base point $G_{0}=(0, y)$ and a point $P_{0}$. We can randomize these points by multiplying random exponents $r, s \in[1, \# E]$, namely let $G=r G_{0}, P=s P_{0}$ be randomized points. From the assumption, we can solve a discrete logarithm problem $\log _{G} P$, and thus the discrete $\log$ arithm $\log _{G_{0}} P_{0}=\left(\log _{G} P\right) r / s \bmod \# E$. 
$(\Rightarrow)$ Let $A_{0}$ be an oracle which solves the discrete logarithm problem for the base point $G_{0}=(0, y)$, namely $A_{0}$ answers $\log _{G_{0}} P_{0}$ for a random point $P_{0}$. We try to construct algorithm $A$ that solves the discrete logarithm problem with a random base. Algorithm $A$ is going to compute $\log _{G} P$ for random inputs $G, P$. Algorithm $A$ randomizes $G$ with a random exponent $t \in[1, \# E]$ and obtains discrete logarithm $\log _{G_{0}} G$ by asking $t G, G_{0}$ to oracle $A_{0}$. Similarly, algorithm $A$ obtains $\log _{G_{0}} P$. Then algorithm $A$ returns the discrete logarithm $\log _{G} P=\left(\log _{G_{0}} P\right) /\left(\log _{G_{0}} G\right) \bmod \# E$.

From this theorem, there is no security disadvantage of using the based point $(0, y)$. Another advantage of using the base point $(0, y)$ is that memory required for base point is reduced to half.

In order to utilize the proposed method efficiently, we propose the following scenario. If we need to resist against both Goubin's attack and the ZVP attack as ECIES and single-pass ECDH, we compute the scalar multiplication on the original curve which has neither Goubin's point $(0, y)$ nor ZVP. Otherwise as ECDSA and two-pass ECDH, we compute on the isogenous curve of a small degree which has a point $(0, y)$, and map the result point to the original curve using isogeny.

We show the example of a curve to achieve this scenario. The curve $E: y^{2}=$ $x^{3}+a x+b$ over $\mathbb{F}_{p}$ defined by

$$
\left\{\begin{array}{l}
p=1461501637330902918203684832716283019653785059327, \\
a=1461501637330902918203684832716283019653785059324=-3, \\
b=650811658836496945486322213172932667970910739301, \\
\# E=14615016373309029182036864189094288558432566759883,
\end{array}\right.
$$

has neither $(0, y)$ nor (ED1) $3 x^{2}+a=0$. Therefore this curve is secure against both Goubin's attack and the ZVP attack for the methods using the standard addition formulae. Then, the 3 -isogenous curve $E^{\prime}: y^{2}=x^{3}+a^{\prime} x+b^{\prime}$ over $\mathbb{F}_{p}$ defined by

$$
\left\{\begin{array}{l}
a^{\prime}=1461501637330902918203684832716283019653785059324=-3, \\
b^{\prime}=457481734813551707109011364830625202028249398260,
\end{array}\right.
$$

has the point $G^{\prime}=(0, y)$ such as

$$
G^{\prime}=(0,914154799534049515652763431190255872227303582054) .
$$

The isogeny $\psi: E \rightarrow E^{\prime}$ and $\psi^{-1}: E^{\prime} \rightarrow E$ cost only $13 M+I$ respectively. This cost is much smaller than improvement of the proposed method. The details of finding such a map are described in [2, Chapter VII].

\section{Conclusion}

We examined the countermeasure using isogeny against the ZVP attack. We showed that a class of curves (including some SECG curves) is still insecure 
against the ZVP attack despite the countermeasure - it can be never mapped to the efficient curve that is secure against the ZVP attack. This class satisfies the following three conditions: $a=-3, E$ has odd order, and $(-3 / p)=-1$. The condition $a=-3$ and $E$ has prime order are important for security or efficiency. Thus the base field $\mathbb{F}_{p}$ with $(-3 / p)=1$ may be recommended.

In the addition, we compare some efficient methods of computing the scalar multiplication for each curve from SECG in consideration of the resistance against the ZVP attack. Finally we proposed a positive use of Goubin's point. If Goubin's point is used for the base point of scalar multiplication, we can improve about $11 \%$ for the double-and-add-always method.

\section{References}

1. T. Akishita and T. Takagi, "Zero-Value Point Attacks on Elliptic Curve Cryptosystem", Information Security - ISC 2003, LNCS 2851, pp. 218-233, Springer-Verlag, 2003.

2. I. Blake, G. Seroussi, and N. Smart, Elliptic Curve in Cryptography, Cambridge University Press, 1999.

3. E. Brier and M. Joye, "Weierstrass Elliptic Curve and Side-Channel Attacks", Public Key Cryptography - PKC 2002, LNCS 2274, pp. 335-345, Springer-Verlag, 2002.

4. E. Brier and M. Joye, "Fast Point Multiplication on Elliptic Curves through Isogenies", Applied Algebra, Algebraic Algorithms and Error-Correcting Codes - AAECC 2003, LNCS 2643, pp. 43-50, Springer-Verlag, 2003.

5. J.-S. Coron, "Resistance against Differential Power Analysis for Elliptic Curve Cryptosystems", Cryptographic Hardware and Embedded Systems - CHES '99, LNCS 1717, pp. 292-302, Springer-Verlag, 1999.

6. W. Fischer, C. Giraud, E. W. Knundsen, and J. -P. Seifert, "Parallel Scalar Multiplication on General Elliptic Curves over $\mathbb{F}_{p}$ Hedged against NonDifferential Side-Channel Attacks", IACR Cryptology ePrint Archive 2002/007. http://eprint.iacr.org/2002/007/

7. L. Goubin, "A Refined Power-Analysis Attack on Elliptic Curve Cryptosystems", Public Key Cryptography - PKC 2003, LNCS 2567, pp. 199-211, Springer-Verlag, 2003.

8. T. Izu, B, Möller, and T. Takagi, "Improved Elliptic Curve Multiplication Methods Resistant against Side Channel Attacks", Progress in Cryptology - INDOCRYPT 2002, LNCS 2551, pp. 296-313, Springer-Verlag, 2002.

9. T. Izu and T. Takagi, "A Fast Parallel Elliptic Curve Multiplication Resistant against Side Channel Attacks", Public Key Cryptography - PKC 2002, LNCS 2274, pp. 280-296, Springer-Verlag, 2002.

10. T. Izu and T. Takagi, "A Fast Parallel Elliptic Curve Multiplication Resistant against Side Channel Attacks", Technical Report CORR 2002-03. http://www.cacr.math.uwaterloo.ca/techreports/2002/corr2002-03.ps

11. M. Joye and C. Tymen, "Protection against Differential Analysis for Elliptic Curve Cryptography", Cryptographic Hardware and Embedded Systems - CHES 2001, LNCS 2162, pp. 377-390, Springer-Verlag, 2001.

12. R. Lercier and F. Morain, "Counting the Number of Points of on Elliptic Curves over Finite Fields: Strategies and Performances", Advances in Cryptology - Eurocrypt '95, LNCS 921, pp.79-94, Springer-Verlag, 1995. 
13. B. Möller, "Securing Elliptic Curve Point Multiplication against Side-Channel Attacks", Information Security - ISC 2001, LNCS 2200, pp.324-334, Springer-Verlag, 2001.

14. B. Möller, "Parallelizable Elliptic Curve Point Multiplication Method with Resistance against Side-Channel Attacks", Information Security - ISC 2002, LNCS 2433, pp.402-413, Springer-Verlag, 2002.

15. P. L. Montgomery, "Speeding the Pollard and Elliptic Curve Methods of Factorization", Mathematics of Computation, vol. 48, pp. 243-264, 1987.

16. K. Okeya and T. Takagi, "The Width-w NAF Method Provides Small Memory and Fast Elliptic Scalar Multiplications Secure against Side Channel Attacks", Cryptographer's Track RSA Conference - CT-RSA 2003, LNCS 2612, pp. 328-343, Springer-Verlag, 2003.

17. N. Smart, "An Analysis of Goubin's Refined Power Analysis Attack", Cryptographic Hardware and Embedded Systems - CHES 2003, LNCS 2779, pp. 281-290, Springer-Verlag, 2003.

18. Standard for Efficient Cryptography (SECG), SEC2: Recommended Elliptic Curve Domain Parameters, Version 1.0, 2000. http://www.secg.org/ 